\title{
Gait retraining as part of the treatment programme for soldiers with exercise-related leg pain: preliminary clinical experiences and retention
}

\author{
W 0 Zimmermann, ${ }^{1,2}$ MD; C W Linschoten, ${ }^{3}$ BSc; A Beutler, ${ }^{2}$ \\ MD \\ ${ }^{1}$ Department of Training Medicine and Training Physiology, Royal Dutch \\ Army, Utrecht, The Netherlands \\ ${ }^{2}$ Uniformed Services University of the Health Sciences, Bethesda, Maryland, \\ USA \\ ${ }^{3}$ Vrije Universiteit, Amsterdam, The Netherlands
}

Corresponding author: W O Zimmermann

(wesselzimmermann@hotmail.com)

Background: Gait retraining as part of a treatment programme for exercise-related leg pain (ERLP) was introduced in the sports medicine department of the Royal Netherlands Army in 2013.

Objectives: To describe clinical experiences and retention of gait retraining in a military setting.

Methods: Sixty-one cases from the year 2015 were available for analysis of gait and gait retraining. In 2016, 32 of these patients were available for a follow-up survey, 28 of them also for the follow-up measurement of running biomechanics in running shoes.

Results: Soldiers received an outpatient treatment programme that lasted on average 129 days (SD 76). On average they received 2.4 gait retraining sessions, leading to significant and lasting changes in running biomechanics; in particular, reduction in maximal force $(\mathrm{N})$ and maximal pressure $\left(\mathrm{N} / \mathrm{cm}^{2}\right)$ on the heels at 317 days follow-up (average, SD 108). Most soldiers were satisfied with gait retraining. At follow-up, 27 soldiers (84\%) contributed some, the majority or all reduction of symptoms to it. Seventy percent reported that they had mastered the new running technique within two months. The Single Assessment Numeric Evaluation score increased from $55 \%$ to $78 \%$ for males and from $44 \%$ to $75 \%$ for females.

Discussion: This is the first study to report on gait retraining for Medial Tibial Stress Syndrome. In future, prospective studies in the military running in shoes and running in boots respectively should be investigated.

Conclusion: Soldiers with exercise-related leg pain (ERLP), among them patients with Medial Tibial Stress Syndrome, respond well to a treatment programme that included gait retraining. Ten months post-gait retraining, their running biomechanics still showed these positive changes from their time of intake.

Keywords: medial tibial stress syndrome, chronic exertional compartment syndrome, military

\section{S Afr J Sports Med 2017;29:1-6. DOI: 10.17159/2078-516X/2017/v29i0a1923}

Exercise-related leg pain (ERLP) is a common problem in the military. Healthy young men can have pain in both legs only weeks after starting a training course, leading to them dropping out of the military. The most common diagnoses in the Armed forces of the Netherlands are: 1. Medial Tibial Stress Syndrome (MTSS), 2. Chronic Exertional Compartment Syndrome (CECS), or 3. A combination of MTSS+CECS.[1] The highest reported incidence of MTSS in a military setting was $35 \%$ of 124 naval recruits participating in basic military training in Australia. ${ }^{[2]}$ An estimation of the incidence of CECS in the military is one in every 2000 US military service members per year. ${ }^{[3]}$

MTSS is an overuse injury involving the interface of the tibial bone and soft tissue. The patient with MTSS reports pain over the tibia during and after leg loading activities. ${ }^{[4]}$ The definition of CECS is pathologically elevated intracompartmental pressure during exercise, which returns to normal with cessation of exercise. ${ }^{[5]}$ The patient with CECS reports a fullness or cramp like sensation over the involved muscular compartment, often after a specific amount of exertion (time, distance, or intensity). ${ }^{[5]}$ If an intracompartmental pressure measurement (ICPM) in the first minute post-exercise is above $35 \mathrm{~mm} \mathrm{Hg}$ and the patient reports pain, the diagnosis CECS is confirmed. ${ }^{[6]}$ If the pressure measurement is below $35 \mathrm{~mm} \mathrm{Hg}$, a new diagnostic term can be applied: Biomechanical Overload Syndrome (BOS)..$^{[7]}$

For both MTSS and CECS, the exact pathophysiological mechanism is not known..$^{[1]} \mathrm{A}$ previous episode of leg pain and the biomechanics of walking and running are a few of many risk factors identified for these conditions in the military. ${ }^{[1]}$ Gait retraining, as a treatment for overuse injuries of the lower extremities is presumably widely practiced but until now, was scarcely reported in the literature. ${ }^{[8]}$ Gait retraining regimens generally focus on a transition from rear foot to midfoot or forefoot strike, increasing cadence, or altering proximal mechanics. ${ }^{[8]}$ The rationale for gait retraining for overuse injuries of the tibia is the reduction of vertical impact forces [9] and for gait retraining in CECS is the reduction of tibialis anterior activity. ${ }^{[10]}$ Gait retraining as a treatment for CECS shows promising results in the first publications on this topic. ${ }^{[11,12]}$ There are no publications on gait retraining as a treatment for MTSS, but recent research indicates its positive effects. ${ }^{[8]}$

In the sports medicine department of the Armed forces of the Netherlands gait retraining as a part of the treatment programme for ERLP was introduced in 2013, using sophisticated tools, such as high-speed cameras and an instrumented treadmill, to analyse walking and running biomechanics. The goal of this study is to evaluate the treatment results and to describe preliminary clinical experiences and the retention of gait retraining in a military setting. This study is retrospective in design.

\section{Methods}

The study involved an analysis of provided patient care 
(patient record analysis), a follow-up survey and measurement of running biomechanics. The inclusion criteria were all soldiers with ERLP who received gait retraining as part of their treatment programme in the year 2015 with the following diagnoses: 1. MTSS; 2. CECS (ICPM > $35 \mathrm{~mm} \mathrm{Hg}$ ); 3. BOS (ICPM < $35 \mathrm{~mm} \mathrm{Hg}) ; 4$. MTSS+BOS; 5. MTSS+CECS. All patients were initially seen by a single, senior sports medicine physician (WZ), using a detailed intake, diagnostic and treatment protocol for ERLP. Exclusion criteria were a fasciotomy less than one year ago and previous gait retraining elsewhere. Minimal follow-up time was at three months.

The following information was retrieved from patient records: patient history, biometrics, pressure measurements of the anterior and deep compartments in the first minute postexercise, diagnosis, kinetics and kinematics of running before gait retraining $\left(\mathrm{T}_{0}\right)$ and after a single gait retraining session on the same day $\left(\mathrm{T}_{1}\right)$.

The gait retraining intervention in 2015 consisted of four instruction sessions: sessions 1 and 4 were given by a primary care sports medicine physician (WZ), and sessions 2 and 3 were given by a physical education instructor. The initial gait retraining session consisted of the following three segments: 1) measurement $\mathrm{T}_{0}$ : one minute of running in running shoes, i.e. personal mechanics; 2) running on bare feet, with verbal instructions to change to ball-of-the foot on landing (when applicable) and preferably 180 steps per minute; 3) measurement $T_{1}$ : one minute of running in shoes, new mechanics. The speed of running was $9 \mathrm{~km} / \mathrm{h}$ for females, 10 $\mathrm{km} / \mathrm{h}$ for males during all running segments and measurements. The running style (type of strike) was determined based on slow-motion camera evaluation and treadmill vertical force measurement. A heel-striker was defined as a visual heel-striker plus a maximum force on the heels $>400 \mathrm{~N}$. During short moments of rest, all participants were shown a video recording of their original and new running mechanics and the measurements of the instrumented treadmill to learn the reduction in impact forces. Instruction sessions 2 and 3 were private gait retraining lessons each lasting 30-60 minutes. All participants received a six-week gait retraining schedule, containing two running sessions a week, to ingrain the new running technique to a continuous running time of 15 minutes. Instruction session 4 , given by the sports physician, was limited to a brief visual check of the new running mechanics. Many patients stayed on for a second sixweek gait retraining schedule, consisting of two-three running sessions a week, to increase running time with the new running technique to 30 minutes at a time. Patients were advised not to run more than the time prescribed in the schedules in order to reduce the chance of a recurrence of symptoms.

Gait retraining was not the only intervention offered in 2015 to patients with ERLP. Each patient received a personalised programme with a mix of the following interventions: stretching or strengthening of lower extremity musculature, supplementation with vitamin D if below $50 \mathrm{nmol} / \mathrm{l}$, massage of hypertonic musculature, dry needling of trigger points, neuro-prolotherapy with $10 \%$ glucose, extra corporeal shockwave therapy of the medial tibial border (four-five sessions), prescription of compression stockings, evaluation of running shoes, evaluation/prescription of shoe inserts, maintaining fitness with a low impact training programme, and radiological imaging. At the end of the treatment programme in the sports medicine department, many patients, particularly those in physically demanding military specialties, were referred to the physical therapist on base for additional training before returning to full duty.

From the telephonic follow-up survey in 2016, the following information was obtained primarily with multiple-choice questions: current military status, current ERLP status, time and effort required to master the new running technique and any additional medical interventions from other medical professionals in the follow-up period.

During the follow-up measurement of running biomechanics $\left(\mathrm{T}_{2}\right)$ the treadmill $(\mathrm{H} / \mathrm{P} / \mathrm{Cosmos}$ Sports \& Medical, Nussdorf, Germany), the software (Zebris Medical, Isny, Germany) and running speed applied were identical to the initial measurements $\left(\mathrm{T}_{0}\right.$ and $\left.\mathrm{T}_{1}\right)$. The treadmill is serviced yearly. The zebris software allowed for immediate feedback on running biomechanics in three zones of the foot: rearfoot, midfoot and forefoot.

In this study, the Single Assessment Numeric Evaluation (SANE) was used as a subjective score for taxability of the legs on a $0-100$ scale, with 100 being normal.[13] The SANE score was recorded at intake (SANE in), at the completion of the sports medicine treatment programme (SANE out) and at follow-up (SANE follow-up).

All statistical tests were performed using SPSS version 24.0. The level of significance was set at $p<0.05$. Data gathered included counts, means and standard deviations for continuous variables and counts and frequencies for categorical variables. A Shapiro-Wilk test was performed to test for normality of the data. If normality was assumed, independent sample t-tests and paired t-tests were conducted; if not, non-parametric testing was performed (Wilcoxon signed ranks test).

The study was announced to the Medical Ethics Board, Brabant, The Netherlands and approved under number NW2016-41.

\section{Results}

In total, 61 cases with ERLP from 2015 were available for record analysis, 48 males and 13 females. Table 1 shows relevant characteristics of these soldiers. The most common diagnoses were MTSS+CECS (20 males and seven females) and MTSS (15 males and five females). The average duration of the treatment programme was 119 days for men $(\mathrm{SD}=63)$ and 176 days for women $(S D=104)$. The average SANE score of patients improved during this time from 54.6 to 78.4 for males and from 44.6 to 75.3 for females. At intake, 52 soldiers were classified as heel-strikers (85\%).

At recall in 2016, 32 patients were available for the follow-up survey $(53 \%)$ (see Figure 1). The average follow-up time was 298 days for men $(\mathrm{SD}=105.2)$ and 357 days for women $(\mathrm{SD}=$ 82.0 ), the average follow-up SANE score was 73.3 for males and 84.5 for females. In addition, 28 of these 32 patients were available to return to the sports medicine department for 
follow-up

treadmill

measurements

(46\%).

Statistically, the soldiers not available for follow-up were no different to the soldiers that were available on the factors presented in Table 1. Reasons for not participating in the follow-up were: no contact possible (17 cases), no time to participate (eight cases) and follow-up time < three months (four cases).

Table 2 shows selected measurements of the running technique in running shoes at $\mathrm{T}_{0}, \mathrm{~T}_{1}$ and $\mathrm{T}_{2}$. Comparison of measurements at $\mathrm{T}_{0}$ and $\mathrm{T}_{1}$ shows that a single session of gait retraining leads to statistically significant changes in most parameters of running measured. The changes in stride length and cadence are relatively small, the changes in force $(\mathrm{N})$ and pressure $\left(\mathrm{N} / \mathrm{cm}^{2}\right)$ on the heels are relatively large. Comparison of measurements at $\mathrm{T}_{0}$ and $\mathrm{T}_{2}$ shows that participants have remained statistically different in most aspects of the running technique measured. For females, the changes in stride length and cadence were no longer statistically different, but force $(\mathrm{N})$ and pressure $\left(\mathrm{N} / \mathrm{cm}^{2}\right)$ on the heels remain significantly reduced at $\mathrm{T}_{2}$ for both males and females. Comparison of measurements at $T_{1}$ and $T_{2}$ shows that males have lost a significant part of their initial reduction of force $(\mathrm{N})$ and pressure $\left(\mathrm{N} / \mathrm{cm}^{2}\right)$ on the heels. At follow-up, seven soldiers were classified as heelstrikers (25\%).

Tables $3 a$ and $3 b$ show information from the follow-up survey. On average, both males and females received 2.4 gait retraining sessions. The number of gait retraining sessions was called 'adequate' by $59 \%$ of the males and $70 \%$ of the females, while the others would have preferred one or two more sessions (Table 3a). Seven patients received only one session (not presented in Table 3a). Most soldiers were positive about gait retraining. At follow-up, 27 soldiers (84\%) contributed some, the majority or all reduction of symptoms to it. Mastering the new running technique was reported to be easy or very easy by 12 soldiers (43\%) and 19 soldiers (70\%) reported that they had mastered the new running technique within two months (Table 3b). After completing the treatment programme in the

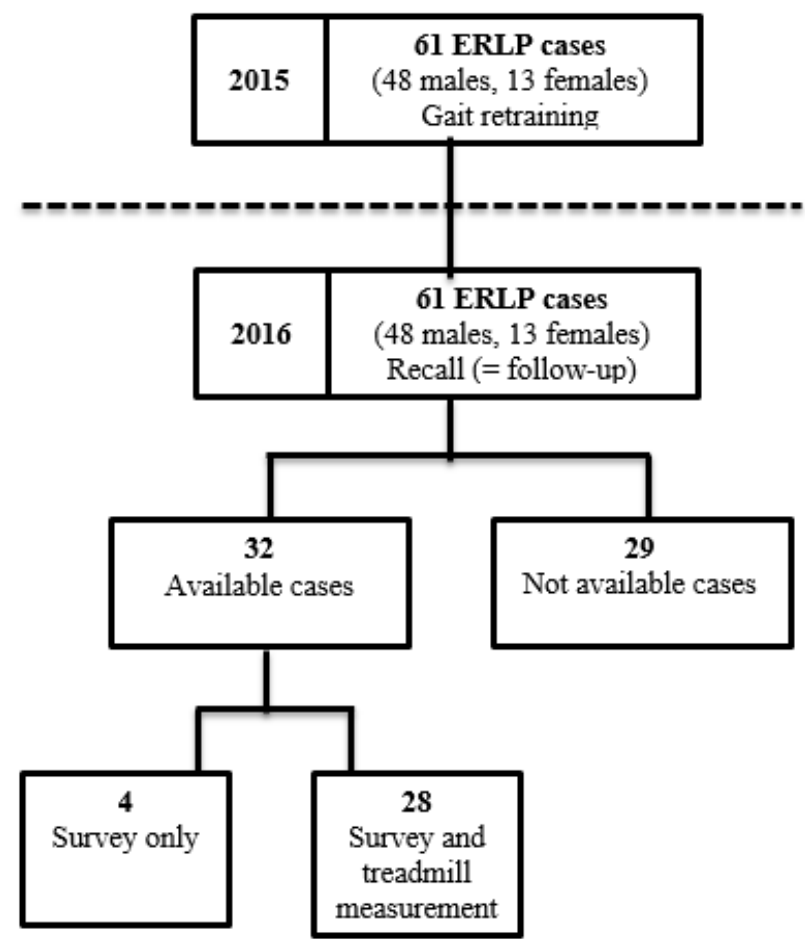

Fig. 1. Study proceedings

sports medicine department, 14 soldiers (44\%) received additional training by a physical therapist and two had surgical treatment (fasciotomy).

Table 4 shows treatment duration, follow-up time and SANE scores in chronological order per diagnostic category. In some 
diagnostic

categories,

already small at intake, only a few participants could be evaluated at follow-up,

therefore no

further statistical calculations were performed on the data in Table 4.

After 129 days of outpatient

treatment and 317

days of follow-up,

military ERLP patients reported an average SANE score of $77 \%$. Patients in the MTSS group had the highest average SANE out scores. Patients in the CECS group had the lowest average SANE out and SANE follow-up scores.

\section{Discussion}

Gait retraining as a treatment for overuse injuries of the lower extremities is presumably widely practiced, but scarcely reported in the literature. This study is a retrospective evaluation of gait retraining offered in 2015 to 61 soldiers with ERLP. Of these soldiers 32 were available for a follow-up survey and of these, 28 for a follow-up $p$ measurement of running technique at 317 days $(S D=108)$. The soldiers not available for follow-up were statistically similar to those who were available. To the best of these authors' knowledge, this is the first study to describe the results of gait retraining for MTSS patients.

At first measurement $85 \%$ of soldiers with ERLP were identified as heel-strikers. This is similar to previous findings on strike patterns among soldiers.[14] One gait retraining session offered by a primary care sports medicine
Table 3a. Information from the follow-up survey

\begin{tabular}{lcc}
\hline & $\begin{array}{c}\text { Male } \\
\mathbf{n = 2 2}\end{array}$ & $\begin{array}{c}\text { Female } \\
\mathbf{n}=\mathbf{1 0}\end{array}$ \\
\hline Currently in military service & $22(100 \%)$ & $9(90 \%)$ \\
Replaced in a lighter specialty & $3(14 \%)$ & $1(10 \%)$ \\
Number of gait retaining sessions received & $2.4 \pm 1.3$ & $2.4 \pm 0.8$ \\
Was this number adequate (yes) & $13(59 \%)$ & $7(70 \%)$ \\
Number of gait retraining sessions preferred & $3.4 \pm 1.5$ & $2.8 \pm 1.2$ \\
\hline
\end{tabular}

Data are expressed as either mean $\pm S D$ or as a count (frequency $\%$ of total).

physician changing strike pattern and introducing relatively small changes in stride length and cadence can produce a statistically significant change in most parameters of running, but, in particular, in maximal force $(\mathrm{N})$ and maximal pressure $\left(\mathrm{N} / \mathrm{cm}^{2}\right)$ on the heels. This reconfirms that the biomechanical parameters of running 
can be readily modified with deliberate instruction. ${ }^{[15]}$ Measurement at follow-up shows that participants lose a percentage of the changes that were made after the first gait retraining session, but a statistically significant reduction in force and pressure at the heels remains (Table 2). At 317 days follow-up $25 \%$ of the soldiers were still heel-strikers, indicating perhaps an individual variation in susceptibility to gait retraining.

At follow-up the average SANE score of ERLP patients was $77 \%$ (Table 4). This shows that many soldiers with ERLP experience persistent difficulty with running even after a comprehensive conservative sports medicine outpatient treatment programme for 317 days. In the Dutch Armed forces, as in the British and American forces, ERLP is a major cause of decreased readiness to continue with training. ${ }^{[1]}$ Continued effort is warranted in both the primary prevention and treatment of these injuries. In previous studies, positive results were reported with gait retraining in the treatment of soldiers diagnosed with CECS. ${ }^{[11,12]}$ In this study patients with MTSS also responded well to a treatment programme, which included gait retraining. This is a novel finding and should encourage healthcare workers to introduce gait retraining as part of the treatment of MTSS patients.

In controlled study settings gait retraining has been executed with eight instruction sessions in two weeks, or 18 sessions in six weeks. ${ }^{[9,11,12]}$ Short-term clinical success with only three gait retraining sessions has been reported. ${ }^{[10]}$ This retrospective analysis shows that some patients had a high SANE follow-up score with as little as a single gait retraining session; however, most soldiers would have preferred three to four sessions. On average, patients received 2.4 gait retraining sessions, where four sessions were intended. Stimulating attendance at all four gait retraining sessions more stringently may improve treatment results in the authors' department and may reduce the number of treatments sought after completing their programme.

This study reports on gait retraining of soldiers running in running shoes. Many patients in this study indicated that their symptoms induced by running in running shoes were enhanced when running in military boots. In the authors' lab, effects of similar magnitude have been observed with gait retraining of running in boots (Meindl, Germany). No studies are available on gait retraining of running in military boots.

This study has several of the inherent limitations of a retrospective analysis: incomplete patient records, different follow-up times per case analysed, patients unavailable for follow-up, and no control group. In addition, patients with different diagnoses in the ERLP group were included and they received different treatment programmes of different duration. It is important to recognise that the benefits of the treatment provided cannot be attributed to gait retraining alone. However, accepting these major limitations, the strength of this study is that it presents new and practical information on gait retraining and its retention as part of a treatment programme for soldiers with ERLP. The followup period, 317 days, is long compared to most published studies ${ }^{[15]}$ and contact with $53 \%$ of the patients, on average after 10 months, is a good recall result in a military setting. It is also an instructive precursor for a prospective study on gait retraining of the same patient population. In future

Table 3b. Patient evaluation of gait retraining at follow-up

\begin{tabular}{lcc}
\hline & Male & Female \\
\hline Time required to master new running technique & $\mathbf{n = 1 9}$ & $\mathbf{n = 8}$ \\
1 month & 11 & 5 \\
2 months & 2 & 1 \\
3 months & 4 & 1 \\
$>3$ months & 2 & 1 \\
Effort required to master new running technique & $\mathbf{n = 2 0}$ & $\mathbf{n = 8}$ \\
Very little, it is very easy & 2 & 0 \\
Little, it is easy & 5 & 5 \\
Intermediate & 7 & 2 \\
A lot, it is hard & 6 & 1 \\
& & \\
Symptom reduction attributed to new running technique & $\mathbf{n}=\mathbf{2 2}$ & $\mathbf{n}=\mathbf{1 0}$ \\
No symptom reduction & 3 & 2 \\
Some symptom reduction & 8 & 3 \\
The majority of symptom reduction & 5 & 2 \\
Complete symptom reduction & 6 & 3 \\
\hline
\end{tabular}

Table 4. Treatment periods and subjective evaluation per diagnostic category

\begin{tabular}{|c|c|c|c|c|c|c|c|c|c|c|c|c|c|c|c|}
\hline & \multicolumn{3}{|c|}{ SANE in (\%) } & \multicolumn{3}{|c|}{$\begin{array}{c}\begin{array}{c}\text { Duration of treatment } \\
\text { (days) }\end{array} \\
\end{array}$} & \multicolumn{3}{|c|}{ SANE out (\%) } & \multicolumn{3}{|c|}{ Follow-up time (days) } & \multicolumn{3}{|c|}{$\begin{array}{c}\text { SANE follow-up } \\
(\%)\end{array}$} \\
\hline & Mean & SD & $\mathbf{n}$ & Mean & SD & $\mathbf{n}$ & Mean & SD & $\mathbf{n}$ & Mean & SD & $\mathbf{n}$ & Mean & SD & $\mathbf{n}$ \\
\hline MTSS & 56 & 18 & 20 & 114 & 43 & 20 & $84^{*}$ & 14 & 15 & 340 & 98 & 12 & $78^{*}+$ & 19 & 12 \\
\hline CECS & 54 & 21 & 5 & 122 & 67 & 4 & 63 & 31 & 3 & 345 & 6 & 2 & 50 & 28 & 2 \\
\hline BOS & 56 & 17 & 5 & 89 & 48 & 5 & $70^{*}$ & 17 & 3 & 287 & 80 & 3 & 80 & 13 & 3 \\
\hline MTSS + BOS & 60 & 10 & 3 & 208 & 135 & 2 & 83 & 4 & 2 & 347 & 263 & 2 & 75 & 35 & 2 \\
\hline MTSS + CECS & 48 & 22 & 27 & 143 & 92 & 27 & $74^{*}$ & 21 & 18 & 294 & 113 & 13 & $80^{*}+$ & 20 & 13 \\
\hline All syndromes & 52 & 20 & 60 & 129 & 76 & 58 & $77^{*}$ & 19 & 41 & 317 & 108 & 32 & $77^{*+}$ & 20 & 32 \\
\hline
\end{tabular}

* Significant change from SANE in at $p<0,05 ;+$ no significant change from SANE out at $p>0,3$

MTSS, Medial Tibial Stress Syndrome; CECS, Chronic Exertional Compartment Syndrome; BOS, Biomechanical Overload Syndrome; Single Assessment Numeric Evaluation (SANE); SANE in, SANE intake; SANE out, completion of the programme; 
studies, it is advisable to measure running mechanics both in running shoes and in military boots at intake and at the completion of the gait retraining intervention. Follow-up measurements could be planned at six and 12 months respectively.

\section{Conclusion}

This study is a retrospective analysis of patient care, with a follow-up, among Dutch soldiers with ERLP. The ERLP patients received on average 2.4 gait retraining sessions. Significant and lasting changes were achieved in running biomechanics, in particular in maximal force $(\mathrm{N})$ and maximal pressure $\left(\mathrm{N} / \mathrm{cm}^{2}\right)$ on the heels at 317 days followup. Soldiers with ERLP were satisfied with gait retraining as part of their treatment programme. Patients with Medial Tibial Stress Syndrome responded well to the treatment programme that included gait retraining as reflected by the increase of their SANE scores. It is suggested that four gait retraining instruction sessions, spread over two-three months, with homework exercises, can be sufficient to produce positive clinical results. In future, prospective studies on gait retraining in the military, both running in running shoes and running in boots should be investigated, because both shod conditions are relevant for the military patient.

Conflict of interest: The authors have no conflict of interest to declare.

Contributions by the authors: Guarantor of this article is WZ. All patients were initially seen by WZ. The follow-up has been executed by CL. Statistics by CL. Text concept by $\mathrm{WZ}$, text contributions by $\mathrm{CL}$ and $\mathrm{AB}$. All authors agree with the final version of this article.

\section{References}

1. Zimmermann WO, Helmhout PH, Beutler A. Prevention and treatment of exercise related leg pain in young soldiers; a review of the literature and current practice in the Dutch Armed Forces. J R Army Med Corps 2017;163:94-103.; [DOI:10.1136/jramc-2016-000635] [PMID:27451420]

2. Yates $B$, White $S$. The incidence and risk factors in the development of MTSS among naval recruits. Am J Sports Med 2004;32(3):772-780. [PMID:15090396];

[DOI:10.1177/0095399703258776]

3. Waterman BR, Liu J, Newcomb R, et al. Risk factors for CECS in a physically active military population. Am J Sports Med 2013;41(11):2545-2549. [DOI:10.1177/0363546513497922] [PMID:23911700]
4. Moen MH, Tol JL, Weir A, et al. Medial tibial stress syndrome: a critical review. Sports Med 2009;39(7):523-546. [DOI:10.2165/00007256-200939070-00002] [PMID:19530750]

5. Rajasekaran S, Hall M. Nonoperative Management of Chronic Exertional Compartment Syndrome: A Systematic Review. Curr Sports Med Rep 2016;15(3):191-198. [DOI:10.1249/JSR.0000000000000261] [PMID:27172084

6. Aweid O, Del Buono A, Malliaras P, et al. Systematic review and recommendations for intracompartimental pressure monitoring in diagnosing chronic exertional compartment syndrome of the leg. Clin J Sport Med 2012;22(4):356-370. [DOI:10.1097/JSM.0b013e3182580e1d] [PMID:22627653]

7. Franklyn-Miller A, Roberts A, Hulse D, et al. Biomechanical overload syndrome: defining a new diagnosis. Br J Sports Med 2014;48(6):415-416. [DOI:10.1136/bjsports-2012-091241] [PMID:22983122]

8. Barton CJ, Bonanno DR, Carr J, et al. Running retraining to treat lower limb injuries: a mixed-methods study of current evidence synthesised with expert opinion. Br J Sports Med 2016;50(9):513-526. [DOI:10.1136/bjsports-2015-095278] [PMID:26884223]

9. Crowell HP, Davis I. Gait retraining to reduce lower extremity loading in runners. Clin Biomech (Bristol, Avon) 2011;26(1):78 83. [DOI:10.1016/j.clinbiomech.2010.09.003] [PMID:20888675]

10. Landreneau LL, Watts K, Heitzman JE, et al. Lower limb muscle activity during forefoot and rearfoot strike running techniques. Int J Sports Phys Ther 2014;9(7),:888-897. [PMID:25540704] [PMCID:PMC4275193]

11. Diebal AR, Gregory R, Alitz C,et al. Forefoot running improves pain and disability associated with chronic exertional compartment syndrome. Am J Sports Med 2012;40(5):1060-1067. [DOI:10.1177/0363546512439182] [PMID:22427621]

12. Helmhout $\mathrm{PH}$, Diebal $\mathrm{AR}$, van der Kaaden $\mathrm{L}$, et al. The Effectiveness Of a 6-Week Intervention Program Aimed at Modifying Running Style in Patients With Chronic Exertional Compartment Syndrome. Orthop J Sports Med 2015;3(3): 11772325967115575691. [DOI:10.1177/2325967115575691] [PMID:26665032]

13. Williams GN, Gangel TJ, Arciero RA, et al. Comparison of the Single Assessment Evaluation Method and two shoulder rating scales: Outcomes measures after shoulder surgery. Am J Sports Med 1999;27(2):214-221. [DOI:10.1177/03635465990270021701] [PMID:10102104]

14. Warr BJ, Fellin RE, Sauer SG, et al. Characterization of FootStrike Patterns: Lack of an Association With Injuries or Performance in Soldiers. Mil Med 2015;180(7):830834.[DOI:10.7205/MILMED-D-14-00220] [PMID:26126256]

15. Napier C, Cochrane CK, Taunton JE, et al. Gait modifications to change lower extremity gait biomechanics in runners: a systematic review. Br J Sports Med 2015;49(12):1382-1388 [DOI:10.1136/bjsports-2014-094393] [PMID:26105016] 